\title{
Minimum Safety Distance Model of Vehicle Under the Influence of Two Factors
}

\author{
Zhang $\mathrm{Yu}$ \\ School of Automotive and Transportation \\ Tianjin University of Technology and Education \\ Tianjin, China
}

\author{
Guan Zhiwei \\ School of Automotive and Transportation \\ Tianjin University of Technology and Education \\ Tianjin, China
}

\begin{abstract}
In the traditional safety distance model, a single factor, such as driver response time or road surface adhesion coefficient, is generally considered. In fact, the safety distance is determined by many factors. Considering the single factor, the safety distance is different from the actual one. Based on previous studies, considering the two factors of driver response time and road adhesion coefficient, the calculation formula of safety distance is re-derived and modeled, and the driver response time is fuzzy inferred by MATLAB. The final model is verified, and a more accurate minimum safety distance is obtained, which has certain reference significance for studying the fluency of traffic flow.
\end{abstract}

Key words-Minimum Safety Distance; Driver Response Time; Road Surface Adhesion Coefficient; Fuzzy Reasoning

At present, China's car ownership is increasing, and it also brings frequent traffic accidents. According to statistics, in China's traffic accidents, rear-end collisions account for about $14 \%$, which is a high-incidence accident. If the distance between cars can be calculated more accurately, rear-end collisions may be greatly reduced.In the intelligent auxiliary driving system, the maintenance of the safe distance is extremely important. The distance can ensure that the vehicle is relatively safe from the front vehicle during longitudinal driving. All along, scholars have studied the safety distance of the workshop, such as the safety distance model based on the headway distance ${ }^{[1]}$, the vehicle anti-collision safety distance model ${ }^{[2]}$, etc. These methods can determine the distance between the two vehicles to some extent. The safety car spacing, which is generally a fixed value. However, there are many shortcomings in practical applications. For example, the change of the driver or the ground adhesion coefficient will affect the driving distance of the vehicle during braking,thus changing the safety distance. This paper will add the driver in combination with the results of previous research. The reaction time and the adhesion coefficient of different road surfaces optimize the model of the vehicle spacing, and finally obtain the safety distance model of different types of drivers when driving on different roads with different adhesion coefficients.

The Key Project of Tianjin Natural Science Foundation of China (Grant No. 16JCZDJC38200)

Tianjin Science and Technology Innovation Platform Project ( 16PTGCCX00150)

\section{ANALYSIS OF THE BRAKING PROCESS}

Under normal circumstances, when the car is bumping in front of the road while driving normally, it is assumed that the speed of the car movement is $v$, the unit is $\mathrm{km} / \mathrm{h}$, and the maximum braking deceleration during braking is $a_{b}$ and the braking phase is for the four stages of $\tau_{1} 、 \tau_{2}, \tau_{3}, \tau_{4}{ }^{[3]}$. As shown in figure 1 :

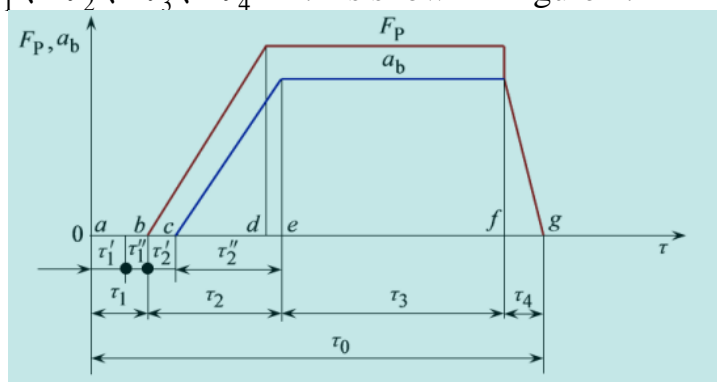

Figure 1 Automotive Braking Process

$\tau_{1}$ is called driver response time. $\tau_{2}$ is called the brake action time. $\tau_{3}$ is called continuous braking time ${ }^{[4]}$.

The time $\tau_{4}$ takes for the driver to release the brake pedal.

In summary, it can be seen that in the normal driving process, considering the driver's reaction time, the distance traveled from the obstacle in front of the vehicle to the time of complete stop is :

$$
s=s_{1}+s_{2}+s_{3}=v \tau_{1}+v \tau_{2}^{\prime}+\frac{v^{2}}{2 a_{\mathrm{b}}}+\frac{v \tau_{2}^{\prime \prime}}{2}-\frac{a_{b} \tau_{2}^{\prime 2}}{24}
$$

\section{RIVER RESPONSE TIME DETERMINATION}

In normal driving behavior, the time during the driver finds the obstacle in front to start braking is called the driver reaction time. There are several situations that affect the driver's reaction time: age and gender, the degree of urgency of the obstacle, the presence or absence of fatigue 
driving, the influence of alcohol or drugs on the driver, the driver's driving mood, and the driver's skill ${ }^{[5]}$.

These factors are qualitative descriptions of driver response time, which is difficult to quantify, so this paper uses fuzzy reasoning to determine the driver's reaction time. In a series of surveys and measurements, the driver's reaction time is between 0.5 and 3 seconds ${ }^{[6]}$, which has a greater impact on the braking distance of the vehicle at high speeds. In this paper, MTALAB is used to perform fuzzy inference on driver response time with a 4-input 1-output mode.

\section{A. Input of fuzzy reasoning}

The four inputs in this paper are the driver's age, driver's driving experience, driver's fatigue level and the degree of urgency of the obstacles in front. Through the driving simulator experiment, Table 1-4 lists the effects of various influencing factors on the reaction time.

\begin{tabular}{cccc}
\multicolumn{4}{c}{ Table 1 Effect of age on reaction time } \\
\hline age & Range & Mean & variance \\
\hline $18-35$ & $1.02-1.8$ & 1.36 & 0.23 \\
$35-55$ & $0.94-2.27$ & 1.89 & 0.21 \\
$55-70$ & $1.5-2.65$ & 2.31 & 0.27 \\
\hline
\end{tabular}

Table 2 Effect of fatigue on reaction time

\begin{tabular}{ccc}
\hline age & $\begin{array}{c}\text { Pre-fatigue } \\
\text { reaction time }\end{array}$ & $\begin{array}{c}\text { Reaction time after } \\
\text { fatigue }\end{array}$ \\
\hline $18-35$ & $0.92-1.03$ & $1.68-1.74$ \\
$35-55$ & $1.06-1.22$ & $2.17-2.32$ \\
$55-70$ & $1.05-1.37$ & $2.35-2.58$ \\
\hline
\end{tabular}

Table 3 Effect of driving age on reaction time

\begin{tabular}{cccc}
\hline $\begin{array}{c}\text { Driving } \\
\text { age }\end{array}$ & $\begin{array}{c}\text { Driver response } \\
\text { time }\end{array}$ & Mean & variance \\
\hline $0-10$ & $0.97-1.39$ & 1.27 & 0.25 \\
$10-30$ & $1.01-1.33$ & 1.19 & 0.36 \\
Over30 & $0.83-1.26$ & 1.04 & 0.29 \\
\hline
\end{tabular}

Table 4 Effect of obstacles on reaction time

\begin{tabular}{cccc}
\hline $\begin{array}{c}\text { The degree of } \\
\text { urgency of the } \\
\text { obstacle }\end{array}$ & $\begin{array}{c}\text { Driver } \\
\text { response time }\end{array}$ & Mean & variance \\
\hline $0-0.3$ & $1.32-1.45$ & 1.37 & 0.31 \\
$0.3-0.7$ & $1.17-1.30$ & 1.26 & 0.27 \\
$0.7-1$ & $0.97-1.25$ & 1.01 & 0.26 \\
\hline
\end{tabular}

In the membership function of driving age, the domain is $[0,50]$, which can be divided into low driving age $j 1$, middle driving age $j 2$, and high driving age $j 3$. In the membership function of age, the domain is [18,70], divided into youth $n 1$, middle-aged $n 2$, and old-aged $n 3$. In the membership function of the obstacles, the degree of urgency of the obstacles is divided into ten levels, that is, the domain is $[0,1]$, which is divided into slow speed $z 1$, moderate speed $z 2$, and fast speed $z 3$. In the membership function of fatigue degree, fatigue is divided into ten grades, that is, the domain is $[0,10]$. Because the degree of fatigue is related to human physiological factors, it is a nonlinear relationship, so Gaussian function is adopted. It is divided into three fuzzy sets with low fatigue degree $p 1$, moderate fatigue degree $p 2$, and high fatigue degree $p 3$. The corresponding membership function is shown in Figure 2.

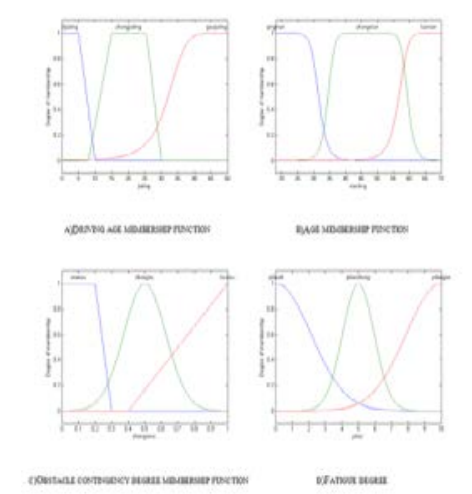

Figure 2 Membership Function Of Four Fuzzy Inputs

\section{B. Output of fuzzy reasoning}

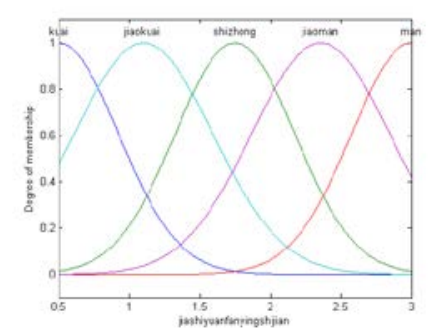

Figure 3 driver reaction time membership function

The driver response time can be determined by the four factors : age, driving age, fatigue level and the degree of urgency of obstacles. The driver response time is within [0.5, 3] and is divided into five ranges, which are Fastest T1. Faster T2, moderate T3, slower T4, slowest T5. A Gaussian membership function is used. The membership function of the driver response time is shown in Figure 3.

\section{Fuzzy rule}

In this paper, the model of 4-input and 1-output is adopted. The "and" connection between different variables is used. The four variables in this paper have three fuzzy sets for each variable, so the corresponding fuzzy rules have $3 \times 3 \times 3 \times 3=81$ rules, as shown in Table 5 . 
Table 5 Driver Response Time Fuzzy Rule Table

\begin{tabular}{cccccccccc}
\hline factor & $n 1 j 1$ & $n 1 j 2$ & $n 1 j 3$ & $n 2 j 1$ & $n 2 j 2$ & $n 2 j 3$ & $n 3 j 1$ & $n 3 j 2$ & $n 3 j 3$ \\
\hline$p 1 z 1$ & $T 2$ & $T 1$ & $T 1$ & $T 2$ & $T 2$ & $T 1$ & $T 3$ & $T 2$ & $T 2$ \\
$p 1 z 2$ & $T 2$ & $T 2$ & $T 1$ & $T 2$ & $T 2$ & $T 1$ & $T 3$ & $T 2$ & $T 2$ \\
$p 1 z 3$ & $T 2$ & $T 1$ & $T 1$ & $T 1$ & $T 1$ & $T 1$ & $T 3$ & $T 2$ & $T 1$ \\
$p 2 z 1$ & $T 3$ & $T 3$ & $T 2$ & $T 4$ & $T 4$ & $T 4$ & $T 4$ & $T 4$ & $T 4$ \\
$p 2 z 2$ & $T 3$ & $T 3$ & $T 3$ & $T 4$ & $T 3$ & $T 3$ & $T 4$ & $T 4$ & $T 4$ \\
$p 2 z 3$ & $T 4$ & $T 2$ & $T 3$ & $T 3$ & $T 3$ & $T 3$ & $T 3$ & $T 3$ & $T 3$ \\
$p 3 z 1$ & $T 4$ & $T 4$ & $T 3$ & $T 5$ & $T 4$ & $T 5$ & $T 5$ & $T 5$ & $T 5$ \\
$p 3 z 2$ & $T 4$ & $T 3$ & $T 3$ & $T 5$ & $T 4$ & $T 5$ & $T 5$ & $T 5$ & $T 5$ \\
$p 3 z 3$ & $T 3$ & $T 3$ & $T 2$ & $T 4$ & $T 3$ & $T 4$ & $T 5$ & $T 4$ & $T 4$
\end{tabular}

After determining the driver's age, driving age, fatigue level, and the degree of urgency of the obstacle, the driver's reaction time can be obtained by defuzzifying in MATLAB.

Figure 4 is a three-dimensional relationship diagram between fuzzy rule input and output.

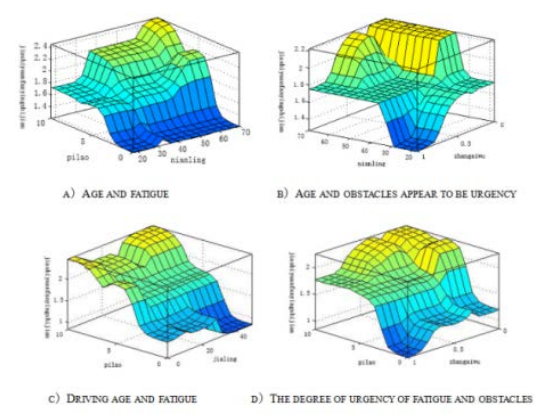

Figure 4 is a three-dimensional relationship between input and output

\section{NFLUENCE OF ROAD ADHESION COEFFICIENT ON BRAKING DISTANCE}

During braking, the brake deceleration directly determines the magnitude of the brake deceleration, which is caused by the friction between the tire and the ground. The amount of friction is determined by the road adhesion coefficient. This paper considers three types of pavement conditions: dry cement pavement, wet slip concrete pavement and ice and snow pavement. Table 6 lists the magnitude of the adhesion coefficient and the maximum brake deceleration for different road surfaces.
Table 6 Size of the adhesion coefficient of different road SURFACES

\begin{tabular}{|c|c|c|c|}
\hline pavement & $\begin{array}{c}\text { Peak } \\
\text { adhesion } \\
\text { coefficient }\end{array}$ & $\begin{array}{c}\text { Sliding } \\
\text { adhesion } \\
\text { coefficient }\end{array}$ & $\begin{array}{c}\text { Maximum } \\
\text { brake } \\
\text { deceleration } \\
\text { (Unit: } \mathrm{m} / \mathrm{s}^{2} \text { ) }\end{array}$ \\
\hline Asphalt & 0.85 & 0.75 & 8.33 \\
\hline asphalt (wet) & 0.6 & 0.5 & 5.88 \\
\hline $\begin{array}{l}\text { Concrete } \\
\text { (wet) }\end{array}$ & 0.8 & 0.7 & 7.84 \\
\hline $\begin{array}{c}\text { Snow } \\
\text { (Press tight) }\end{array}$ & 0.2 & 0.15 & 1.96 \\
\hline ice & 0.1 & 0.07 & 0.98 \\
\hline
\end{tabular}

In an emergency, the driver slams on the brakes and the vehicle is fully braked. Because of the ABS, the ground adhesion coefficient can reach the peak adhesion coefficient, and the vehicle can generate the maximum braking deceleration. Let the maximum ground braking force of the vehicle be $F_{X b \max }$, the normal reaction force of the ground to the wheel is $F_{Z}$, and the road surface adhesion coefficient is $\varphi$ : the relationship between the three is:

$$
F_{X b \max }=F_{z} \varphi
$$

The maximum braking force of the vehicle on different road surfaces is obtained by the above formula, and the braking deceleration on different road surfaces can be obtained.

Assuming that the mass of the vehicle is $m$, the acceleration of gravity is $g$, then the maximum brake deceleration of the vehicle during braking can be obtained.

$$
F_{x b \max }=m g \varphi
$$

Deduced

$$
a_{\max }=\frac{F_{X b \max }}{m} g \varphi
$$

It can be known from formula (4) that $a_{\max }$ is related to the road surface adhesion coefficient. 
IV. ESTABLISHMENT OF A MATHEMATICAL MODEL

\section{OF MINIMUM SAFE DISTANCE}

The establishment of the minimum safety distance model helps to improve the patency and efficiency of traffic on the basis of safety. This paper mainly considers two aspects: the uniform speed of the front car and the uniform deceleration of the front car. Which will be discussed separately.

\section{A. Front car uniform speed}

Figure 5 shows the motion of the two cars in front and rear.

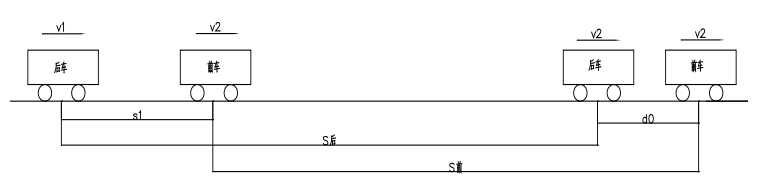

Figure 5 Schematic diagram of vehicle movement before and after

As shown in the figure, the front car runs at a constant speed, and the rear car speed is greater than that of the preceding car. In this case, if the rear car does not brake, it will collide with the preceding car at some later time, which is dangerous . Therefore, the rear vehicle should be decelerated when it is at a certain distance from the preceding vehicle (ie, the minimum safe distance), and decelerating to the same speed as the preceding vehicle is a safe working condition.

It is assumed that the distance between the two vehicles is $S_{1}$ at the beginning, and when the speed of the rear vehicle is the same as that of the preceding vehicle, the distance traveled by the rear vehicle is $S_{f}$, and the distance traveled by the preceding vehicle is $S_{r}$,

$$
S_{1}=S_{r}-S_{f}+d_{0}
$$

That is, the minimum safe distance is obtained in this paper, where $d_{0}$ is the distance between the rear and front vehicles and the speed of them are same, generally take $d_{0}=5 \mathrm{~m}$

The front car moves at a constant speed, and the rear car speed is greater than the front one. According to the speed difference between the front and rear cars, it can be divided into three situations:

(1) When the brake deceleration is in the growth phase, the vehicle speed is the same.

The distance traveled by the front car is

$$
S_{f}=v_{2}\left(t_{0}+t_{1}+t_{1}^{\prime}+t_{2}^{\prime}\right)
$$

The distance traveled by the rear car is

$$
S_{r}=v_{1} t_{0}+v_{1} t_{1}+t_{1}^{\prime}+v_{1} t_{2}^{\prime}-\frac{1}{6} a_{1 \max } \frac{t_{2}^{\prime}}{t_{2}} t_{2}^{2}
$$

Minimum safe distance

$$
S_{1}=S_{r}-S_{f}+d_{0}=\left(v_{1}-v_{2}\right)\left(t_{0}+t_{1}+t_{1}^{\prime}+t_{2}^{\prime}\right)-\frac{1}{6} a_{1 \max } \frac{t_{2}^{\prime}}{t_{2}} t_{2}^{2}+d_{0}
$$

The meaning of each letter in the formula :

$v_{1}$ rear car speed; $v_{2}$ front car speed; $t_{0}$ to eliminate the brake system clearance time ; $t_{1}$ driver response time ; $t_{1}$ the time it takes for the driver to put his foot on the brake pedal ; $t_{2}$ deceleration of the vehicle to the same speed as the vehicle speed ; $a_{1 \max }$ the maximum brake deceleration of the rear car.

(2) The vehicle speed is the same when the brake deceleration just increases to the maximum brake deceleration.

In this case, the driving distance of the preceding vehicle is

$$
S_{f}=v_{2}\left(t_{0}+t_{1}+t_{1}^{\prime}+t_{2}\right)
$$

The distance traveled by the rear car is

$$
S_{r}=v_{1} t_{0}+v_{1} t_{1}+v_{1} t_{1}+v_{1} t_{2}-\frac{1}{6} a_{1 \max } t_{2}^{2}
$$

Minimum safe distance:

$$
\begin{aligned}
& S_{1}=S_{r}-S_{f}+d_{0} \\
& =\left(v_{1}-v_{2}\right)\left(t_{0}+t_{1}+t_{1}+t_{2}\right)-\frac{1}{6} a_{1 \max } t_{2}^{2}+d_{0}
\end{aligned}
$$

In the formula, $t_{2}$ is the vehicle acceleration growth time.

(3) The vehicles speed is the same while the brake deceleration increases to the maximum brake deceleration and continues to brake for a while.

In this case, the distance traveled by the preceding vehicle is :

$$
S_{f}=v_{2}\left(t_{0}+t_{1}+t_{1}^{\prime}+t_{2}+t_{3}\right)
$$

The distance traveled by the rear car is : 


$$
\begin{aligned}
& S_{r}= \\
& v_{1} t_{0}+v_{1} t_{1}+v_{1} t_{1}^{\prime}+v_{1} t_{2}-\frac{1}{6} a_{1 \max } t_{2}^{2}+\frac{\left(v_{1}-\frac{1}{2} a_{1 \max } t_{2}\right)^{2}-v_{2}^{2}}{2 a_{1 \max }}
\end{aligned}
$$

Then, the minimum safe distance sought :

$$
\begin{aligned}
& S_{1}=S_{r}-S_{f}+d_{0}= \\
& v_{1}\left(t_{0}+t_{1}+t_{1}^{\prime}+t_{2}\right)-\frac{1}{6} a_{1 \max } t_{2}^{2}+\frac{\left(v_{1}-\frac{1}{2} a_{1 \max } t_{2}\right)^{2}-v_{2}^{2}}{2 a_{1 \max }}-v_{2}\left(t_{0}+t_{1}+t_{1}^{\prime}+t_{2}+t_{3}\right)+d_{0}
\end{aligned}
$$

The time when the rear car continues to brake is :

$$
t_{3}=\frac{v_{1}-v_{2}}{a_{1 \max }}-\frac{1}{2} t_{2}
$$

\section{B. Front car non-uniform motion}

Under such conditions, the change of the speed of the preceding vehicle becomes more and more complicated, and the corresponding braking operation of the rear vehicle is based on the response of the preceding vehicle's motion state. In order to ensure that it does not collide with the preceding car, this paper considers three situations under the premise of uniform deceleration of the preceding vehicle. The motion diagram of the front car under uniform deceleration is shown in Figure 6.

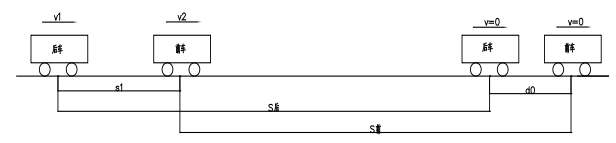

Figure 6 Schematic diagram of uniform deceleration movement of the front car

The speed of the front car is greater than the speed of the rear one .

The front car is decelerated and moved at a certain braking deceleration until it stops. In this case, the calculated minimum safety distance is the smallest. As long as the rear car and the front car ensure this safe distance, it will not be collision and because of the small safety distance, it can also ensure the smoothness and high efficiency of traffic flow.

Set the front car speed is $v_{2}$, The deceleration of the front car is $a_{2}$, Rear car speed is $v_{1}$, The maximum brake deceleration of the rear car is $a_{1 \max }, d_{0}$ is $5 \mathrm{~m}$.The displacement of the preceding vehicle from $v_{2}$ deceleration $a_{2}$ to stop is

$$
S_{f}=\frac{v_{2}^{2}}{2 a_{2}}
$$

After the car found the front car decelerating, the time taken to eliminate the brake system clearance is $t_{0}$,
Driver response time is $t_{1}$, The time it takes the driver to put his foot on the brake pedal is $t_{1}^{\prime}$, Brake deceleration growth time is $t_{2}$, Then the distance traveled by the rear car is :

$$
S_{r}=v_{1} t_{0}+v_{1} t_{1}+v_{1} t_{1}^{\prime}+v_{1} t_{2}+\frac{v_{1}^{2}}{2 a_{1 \max }}-\frac{v_{1} t_{2}}{2}-\frac{a_{1 \max } t_{2}^{2}}{24}
$$

Then the minimum safe distance is

$$
\begin{aligned}
& S_{1}=S_{r}-S_{f}+d_{0}= \\
& v_{1} t_{0}+v_{1} t_{1}+v_{1} t_{1}^{\prime}+v_{1} t_{2}+\frac{v_{1}^{2}}{2 a_{1 \max }}-\frac{v_{1} t_{2}}{2}-\frac{a_{1 \max } t_{2}^{2}}{24}-\frac{v_{2}^{2}}{2 a_{2}}+d_{0}
\end{aligned}
$$

(2) The speed of the front and rear cars is the same

This situation is similar to the previous one. Simply change the formula (18) slightly and the minimum safe distance is:

$$
\begin{aligned}
& S_{1}=S_{r}-S_{f}+d_{0}= \\
& v_{1} t_{0}+v_{1} t_{1}+v_{1} t_{1}^{\prime}+v_{1} t_{2}+v_{1}^{2}\left(\frac{1}{2 a_{1 \max }}-\frac{1}{2 a_{2}}\right)-\frac{v_{1} t_{2}}{2}-\frac{a_{1 \max } t_{2}^{2}}{24}+d_{0}
\end{aligned}
$$

(3) The speed of the front car is greater than the speed of the rear car

In this case, if the front car has been decelerating and the rear car is not processed, there will be dangerous after driving for a period of time. Therefore, the rear car should be braked to stop when the preceding vehicle speed is reduced to the same as the rear one. The distance traveled by the preceding car during this process is:

$$
S_{f}=\frac{v_{2}^{2}}{2 a_{2}}
$$

The distance traveled by the rear car is:

$$
S_{r}=v_{1} \frac{v_{2}-v_{1}}{a_{2}}+v_{1} t_{0}+v_{1} t_{1}+v_{1} t_{1}^{\prime}+v_{1} t_{2}-\frac{v_{1} t_{2}}{2}-\frac{a_{1 \max } t_{2}^{2}}{24}+d_{0}
$$

Then the minimum safe distance is:

$$
S_{1}=S_{r}-S_{f}+d_{0}=v_{1}\left(t_{0}+t_{1}+t_{1}^{\prime}+\frac{t_{2}}{2}\right)+\frac{v_{2}^{2}}{2 a_{2}}-\frac{v_{1}^{2}}{a_{2}}-\frac{a_{1 \max } t_{2}^{2}}{24}+d_{0}
$$

\section{Selection of parameters}

Before the simulation, it is necessary to determine the 
value of some physical quantities. From the above analysis, it can be seen that in the situation of uniform motion of the front vehicle and rear car deceleration movement, the time $t_{0}$ which taken to overcome the gap of the braking system are same.This paper sets $t_{0}$ value range 0.05-0.1 s the same time $t_{2}$ from the start of the brake deceleration to the front and rear speeds and $t_{2}$ seconds from the start of the brake deceleration to the maximum of the brake deceleration. The value range of this paper $t_{2}$ is set to $0.15-0.3 \mathrm{~s}$. The determination of $t_{2}^{\prime}$ is related to the deceleration of the front and rear vehicles before and after the vehicle speed, and is analyzed in detail when doing the analysis.

\section{V.MODEL SIMULATION ANALYSIS}

This paper uses the Matlab platform to simulate the model. The deceleration values of the front and rear brakes under different road adhesion factors are shown in the following table:

Table 7: Deceleration of front and rear brakes under different road surface adhesion coefficients

\begin{tabular}{lccc}
\hline & & $\varphi$ & \\
\cline { 2 - 4 } & 0.85 & 0.6 & 0.2 \\
\hline$a_{1 \text { max }}$ & 8.33 & 5.88 & 1.96 \\
$a_{2}$ & 5 & & \\
& & 3 & 1 \\
\hline
\end{tabular}

\section{A. Front car uniform motion}

The simulation of the front car under uniform motion is shown in Figure 7-10.

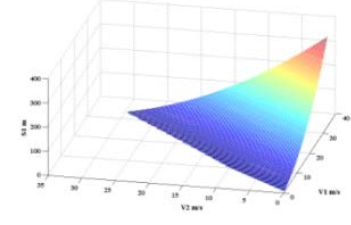

FIGURE 7 REACTION TMME $2.66 \mathrm{~S} \varphi=0.2$

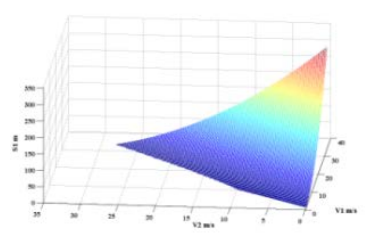

FigURE 9 REACTION TIME $0.88 \varphi=0.2$

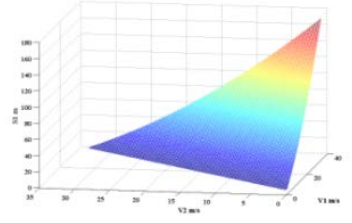

FIGURE 8 REACTION TIME $2.665 \quad \varphi=0.85$

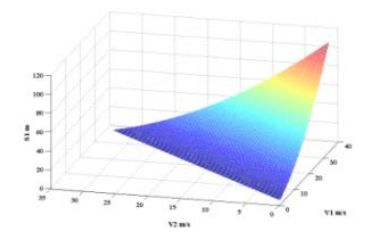

FIGURE 10 REACTION TME $0.886 \varphi=0.85$

\section{FIGURE 7TO10}

It can be seen from figs. 7 to 8 that under the same reaction time, the influence of the road surface adhesion coefficient on the braking distance of the vehicle is very high. In the case of the small road surface adhesion coefficient, the maximum braking distance is almost 400 meters, and the braking distance in the peak value of the road surface adhesion coefficient has not reached 150 meters.

As can be seen from figs. 7 and 9, while the road surface adhesion coefficient is same, the longer the driver reaction time is, the larger the braking distance is.

\section{B. Front car uniform deceleration}

As shown in Figure 11 to 14, in the case of uniform deceleration of the preceding vehicle, the influence of the driver's reaction time and the road surface adhesion coefficient on the braking distance of the vehicle are similar to that of the preceding vehicle. The speed range is small at the figure 12 figure 14 , because if the speed of the front car is greater than a certain value, the distance from the front car to the stop is far longer than the distance from the rear car to the speed of the deceleration, so take The range of values will be smaller.

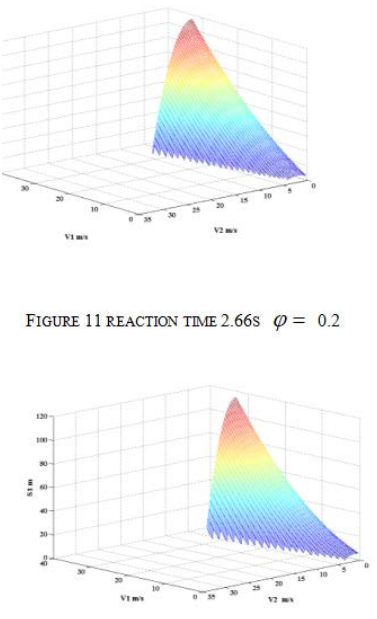

FIGURE 13 REACTION TIME0.88 $\varphi=0.2$

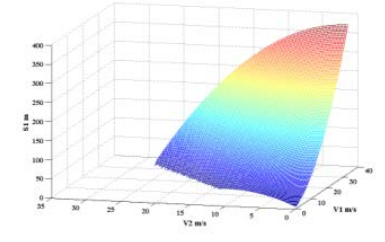

FIGURE 12REACTION TIME $2.66 \mathrm{~S} \varphi=0.85$

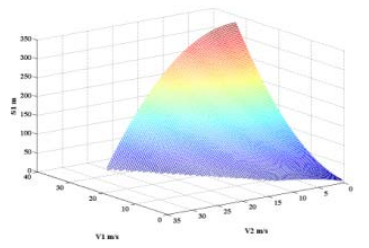

FIGURE 14 REACTION TMME $0.886 \varphi=0.85$
Figure 11to14

It can be seen from the above analysis that different driver response time and road surface adhesion coefficient have a certain degree of influence on the braking distance. In the design of intelligent auxiliary driving,different driving distances shuold be judgment and adjustment, according to different drivers and different road surface adhesion coefficients.

\section{VI.CONCLUSION}

In the daily driving process, different drivers have different reaction times, and different road adhesion coefficients have different braking deceleration. These two factors are important factors for calculating the safety distance through the above simulation analysis. In the case of a certain speed of front and rear vehicles, the minimum safety distance is obtained by 
different drivers and different road surface adhesion coefficients is not same. If the driver's monitoring and the road adhesion coefficient are added to the intelligent auxiliary drive system, it is of great significance in preventing the occurrence of rear-end collision accidents, and it is superior to the traditional model in improving road traffic efficiency.

\section{REFERENCES}

[1] Wang Jiangfeng, Shao Chunfu, Yan Xuedong, Wei Liying. Research on Minimum Safe Distance of Vehicle Lane Change Based on Virtual Reality[J].Highway transportation technology,2010,27(8):109-113.

[2] Luo Qiang, Xu Lunhui. Establishment and Simulation of Car-following odel Based on Minimum Safety Distance[J].Science and technology and engineering,2010,10(2):569-573.

[3] Wang Junlei, Li Baichuan, Ying Shijie, Gong Hangjun.A Study on Collision Early Warning Analysis and Minimum Vertical Safety Distance Model of Lane Change[J].Ergonomics,2004(4):16-19.

[4] Lu Jier, Zhu Liuhua, Zheng Rongsen, Wei Yanfang. The Impact of Driver Response Time on Traffic Safety[J].Transportation System Engineering and Information,2014,14(2):80-86.

[5] Critical safe distance design to improve driving safety based on vehicle-to-vehicle communications [J] . Journal of Central South University,2013,20(11):3334-3344.

[6] C.C.Yuan. Analysis the safety distance model and modeling DRV safety distance model[A]. IETP Associaiton.Abstract of the 2015 International Conference on Advanced Materials and Engineering Structural Technology(ICAMEST 2015)[C].IETP Associaiton:,2015:1. 